\title{
RNA-silencing induces target gene relocalization toward a specialized nuage domain
}

Yuchen Yang ${ }^{1,}$, David Grunwald ${ }^{1,}$, James R. Priess, ${ }^{2,3}$ and Craig C. Mello ${ }^{1,4,5^{*}}$

\section{Author Affiliations:}

1. RNA Therapeutics Institute, University of Massachusetts Medical School, 9 Worcester, MA 01605

10 2. Fred Hutchinson Cancer Research Center, Seattle, Washington, United States

11 3. Department of Biology, University of Washington, Seattle, Washington, United States

12 4. Program in Molecular Medicine, University of Massachusetts Medical School, 368

13 Plantation Street, Worcester, MA, 01605 United States

14 5. Howard Hughes Medical Instifute, University of Massachusetts Medical School,

15 Worcester, MA, USA

16 * Correspondence, Craig.Mello@umassmed.edu. 
17 Abstract

18 Argonaute small RNA pathways maintain genome integrity and fertility by

19 enforcing the transgenerational silencing of transposons as well as many

20 developmentally regulated germline genes. To propagate silencing, Argonaute

21 pathways coordinate heterochromatin silencing with cycles of small RNA

22 amplification. In animal germlines, mRNA surveillance is thought to occur within

23 cytoplasmic perinuclear domains called nuage. In C. elegans, nuage granules

24 called $\mathbf{P}$ granules surround each pachytene germline nucleus. $\mathbf{P}$ granules are

25 known to host many of the Argonaute small RNA systems that carry out

26 transcriptome surveillance, but what if any specific roles $\mathbf{P}$ granules might play in

27 Argonaute silencing have remained mysterious. Here we show that RNAi triggers

28 the enlargement of a single P granule, which uniquely accumulates target RNA. P

29 granule enlargement occurs independently of, and precedes transcriptional

30 silencing, which culminates in the relocalization of both alleles of the target gene

31 to the inner nuclear membrane (INM) directly adjacent the specialized P granule.

32 Similarly, we show that piRNA-mediated silencing, causes both alleles of a targeted

33 gene to reside near the INM adjacent to a $\mathbf{P}$ granule that uniquely contains target

34 RNA sequences. Our findings suggest that nuage and its associated Argonaute

35 pathways re-organize the three dimensional location of their genomic targets to

36 facilitate RNA-guided inheritance. 


\section{Introduction}

41 In the $C$. elegans germline, two major Argonaute pathways initiated by different

42 mechanisms - the piRNA pathway(Watanabe and Lin, 2014) mediated by the Piwi

43 Argonuate PRG-1 and the dsRNA-initiated RNAi pathway (Fire et al., 1998) mediated by

44 the Argonaute RDE-1 (Chen et al., 2005)-rely on a common set of conserved

45 downstream small RNA and chromatin effectors that maintain heritable silencing (Billi et

46 al., 2014; Holoch and Moazed, 2015; Weiser and Kim, 2019). Argonaute proteins and the

47 machinery that produces their small RNA cofactors localize to P granules (Updike and

48 Strome, 2010), where they are thought to act in mRNA surveillance and in the

49 amplification of small-RNA silencing signals (Billi et al., 2014; Weiser and Kim, 2019). In

50 the C. elegans meiotic germline, each $\mathrm{P}$ granule is localized over a cluster of nuclear

51 pores (Pitt et al., 2000), where it has the potential to intercept nascent mRNA en route to

52 the cytoplasm (Sheth et al., 2010). Although previous cytological studies demonstrated

53 the rapid loss of target mRNA in the cytoplasm during RNAi (Fire et al., 1998; Sheth et

54 al., 2010), changes in the $\mathrm{P}$ granules or associated RNAs during silencing were not

55 reported and the localization of target DNA was not examined. Thus, the dynamics and

56 spatial relationships between nuage and the mRNA and DNA targets of silencing have

57 remained mysterious.

Recent advances in RNA and DNA FISH technology have greatly enhanced the

59 ability to track molecules at single-molecule resolution (Beliveau et al., 2015; Fields et al.,

60 2019; Ji and van Oudenaarden, 2012). For example, splinted fluorescent probes that

61 assemble multiple fluorophores at a single target greatly amplify DNA FISH signals and

62 permit detection of single genes(Beliveau et al., 2015; Fields et al., 2019). Here we utilize 
63 these improved reagents to revisit the cytological events associated with Argonaute

64 silencing.

\section{Results and Discussion}

67 To visualize the spatial and temporal response to RNAi, we exposed worms to dsRNA

68 targeting an abundantly transcribed germline-expressed gene, oma-1, and monitored

69 oma-1 RNA- and DNA-FISH signals simultaneously over a time course of silencing. To

70 distinguish cytoplasmic and nuclear signals in RNA + DNA FISH experiments, we marked

71 the outer membrane of the nuclear envelope with a GFP:ZYG-12 fusion protein (Malone

72 et al., 2003) (Figure 1A-1F), while P granules were identified with GFP::GLH-1 (Figure

73 1G-1L) tagged at the endogenous glh-1 locus by CRISPR genome editing (Ghanta et al.,

74 2020). We examined the pachytene region of the gonad, where germ cells are connected

75 to a large, shared region of cytoplasm called the core. As expected from previous studies,

76 oma-1 mRNA in untreated animals was present at low levels in many $\mathrm{P}$ granules, and

77 was abundant in the germ cell cytoplasm and gonad core (Figure 1J). A double dot of

78 oma-1 RNA signal was visible inside each germ nucleus throughout the pachytene region

79 (Figure 1A, 1D and 1J). The nuclear RNA dots co-localized with oma-1 DNA in

80 simultaneous RNA/DNA FISH experiments (Figure 1A), suggesting that they represent

81 transcription from the oma-1 genes on the paired, homologous chromosomes.

82 As expected, RNAi induced a rapid, marked depletion of cytoplasmic oma-1 mRNA

83 (for example, compare Figure 1A to 1B). In the same gonads, however, an oma-1 RNA

84 signal appeared to increase within a single large, perinuclear focus adjacent to each 
85 nucleus (Figure 1B and 1E). This perinuclear RNA signal was also present at 21 hours 86 but was decreased in size (Figure $1 \mathrm{C}$ and $1 \mathrm{~F}$ ).

87 Prior to RNAi, the P granules varied in size (green signal in Figures $1 \mathrm{G}$ and $1 \mathrm{~J}$ ).

88 However, after 6 hours of RNAi, one P granule on each nucleus appeared much larger

89 than neighboring nuage (Figure $1 \mathrm{H}$ ), and colocalized with the prominent, enlarged oma-

901 RNA focus (Figure 1K). Thus, RNAi appears to trigger differentiation between the $\mathrm{P}$

91 granules on the same nucleus, with target RNA accumulating in only one enlarged $\mathrm{P}$

92 granule. These results suggest that a single $\mathrm{P}$ granule becomes specialized for oma-1

93 silencing.

94 The location of the oma-1 RNA and DNA FISH signals within the nucleus exhibited

95 dramatic changes during RNAi. Whereas prior to RNAi each pachytene nucleus exhibited

96 two paired foci of DNA and the coincident RNA (Figure 1A), by 21 hours nuclei exhibited

97 only a single focus of oma-1 DNA and a coincident RNA signal (Figure 1C). These

98 coincident signals were located near the INM (Figure 1F) and directly adjacent to the

99 specialized $\mathrm{P}$ granule which, although now reduced in size, remained the only $\mathrm{P}$ granule

100 that contained oma-1 RNA (Figure 1I and 1L). The transition to a single focus of oma-1

101 DNA and coincident RNA was evident in many nuclei by 6 hrs of oma-1(RNAi) (Figure

102 1B, inset ii), and when present this singular focus was always positioned near the INM

103 adjacent the specialized $\mathrm{P}$ granule (Figure $1 \mathrm{~K}$, insets $\mathrm{i}$ and ii). Taken together these

104 results suggest that RNAi causes the formation of a unique enlarged $\mathrm{P}$ granule where

105 target RNA accumulates, and that formation of this specialized P granule precedes and

106 possibly coordinates movement of the target DNA to the directly adjacent INM. 
In order to determine whether genes in the RNAi pathway are required for the specialized $\mathrm{P}$ granule, we repeated the above assays in the mutant $r d e-3$. RDE-3 is an

109 effector of RNAi silencing that is required both in the animals exposed to dsRNA and for

110 inherited silencing among their offspring (Chen et al., 2005). As expected, in rde-3

111 mutants oma-1 dsRNA did not trigger any of the cytological changes observed in wild-

112 type worms (compare Figures 2A and 2B with Figures 2C and 2D, respectively). For

113 example, the double dots of oma-1 DNA did not relocalize toward the INM, low levels of

114 oma-1 RNA remained visible in many $\mathrm{P}$ granules, which appeared similar in size to those

115 in untreated controls, and cytoplasmic oma-1 mRNA remained abundant in the gonad

116 core (Figure 2A and 2B and Supplemental Figure 2).

117 Nuclear effectors of RNAi are known to drive heterochromatin formation and

118 several studies have linked heterochromatin formation to the localization of a silent gene

119 near the INM (Reviewed in (Ahringer and Gasser, 2018)). We therefore wondered

120 whether the nuclear effectors of the RNAi pathway are required for relocalization of the

121 target genes, and whether targeting events at nuclear transcription sites might precede

122 and drive $\mathrm{P}$ granule specialization. To explore these possibilities we examined the role of

123 two genes, the nuclear WAGO Argonaute, wago-9/hrde-1 and its downstream effector

124 nrde-2. These genes are both required for establishment of heterochromatin at the target

125 locus and for transgenerational inheritance of silencing induced by RNAi (Ashe et al.,

126 2012; Buckley et al., 2012; Burton et al., 2011; Guang et al., 2010; Shirayama et al., 2012).

127 Importantly, neither gene is essential for RNAi when a dsRNA trigger is present, but rather

128 are only required for transmission of silencing to unexposed offspring. dsRNA triggered

129 the loss of cytoplasmic oma-1 mRNA in both mutants, simlar to wild type, and the 
130 accumulation of oma-1 RNA in a single, enlarged $\mathrm{P}$ granule (Compare Figures 2E and

$1312 \mathrm{G}$ with Figure 2C). However, the nuclei retained two separate foci of DNA and coincident

132 RNA that did not relocalize toward the INM even after 21 hours of RNAi (compare Figure

$1332 \mathrm{~F}$ and $2 \mathrm{H}$ to Figure 2D). Moreover, by $21 \mathrm{hrs}$, the unique enlarged $\mathrm{P}$ granule had

134 disappeared and oma-1 RNA was detected in multiple smaller $\mathrm{P}$ granules (Fig 2F and

$1352 \mathrm{H}$ ). Thus the nuclear events of RNAi are not required for the initial formation of the

136 specialized $\mathrm{P}$ granule, but instead function to promote its maintenance and to coordinate

137 the nuclear relocalization of the target genes into its proximity.

138 The C. elegans germline expresses tens of thousands of piRNAs that engage Piwi

139 Argonautes to direct transgenerational silencing of transposons and many protein-coding

140 genes (Billi et al., 2014). We wondered if piRNA silencing might also direct the

141 coordination of the target loci and a specialized P granule. To address this possibility we

142 examined the dosage compensation gene $x o l-1$, which is a natural target of piRNA

143 silencing (Tang et al., 2018). Mutations in the Piwi Argonaute, prg-1, cause xol-1

144 expression in the germline and lead to dosage-compensation defects among progeny.

145 We first examined prg-1 mutants to determine where $x o l-1$ is expressed when de-

146 repressed. Although xol-1 was expressed at lower levels than oma-1, we found that late

147 pachytene nuclei in prg-1 mutants had a pattern of $x o l-1$ DNA and RNA signals similar to

148 the pattern seen for oma-1 in wild-type animals prior to dsRNA exposure. For example,

149 the nuclei each contained two foci of $x o l-1$ DNA and coincident RNA, and xol-1 mRNA

150 was visible in several perinuclear $P$ granules (Figures $3 A$ and $3 B$ ). In contrast, late

151 pachytene germ cells in wild-type animals, where xol-1 is repressed, exhibited a pattern

152 of $x o l-1$ DNA and RNA FISH signals similar to that of silenced oma-1 after 21 hours of 
153 RNAi (Figure 3C and 3D). For example, the xol-1 genes appeared as a single dot

154 coincident with the xol-1 nuclear RNA signal, and were localized adjacent to a P granule

155 enriched for $x o l-1$ RNA. Thus, the cytology of the PRG-1-dependent OFF state of $x o l-1$ in

156 wild-type animals resembles the dsRNA-induced OFF state of a gene targeted by RNAi.

157 We wondered whether different $\mathrm{P}$ granules become specialized to silence distinct

158 targets. To address this possibility we decided to monitor both oma-1 and xol-1

159 simultaneously in wild-type animals over a time course of oma-1(RNAi). The oma-1 and

160 xol-1 genes are on different chromosomes, and their respective nuclear signals of DNA

161 and RNA generally remained well separated over the time course of RNAi (Figure 3E and

$1623 \mathrm{~F}$ ). For example at $6 \mathrm{hr}$ of RNAi the enlarged, specialized $\mathrm{P}$ granule associated with

163 oma-1 silencing formed independently of the nuclear transcription site of xol-1 and its

164 associated $\mathrm{P}$ granule (Figure 3E), and at $21 \mathrm{hr}$ of RNAi the oma-1 genes were positioned

165 near the INM independently of the xol-1 DNA (Figure 3F). Together these findings

166 suggest that each $\mathrm{P}$ granule promotes silencing of distinct genomic targets, and that the

167 three dimensional positioning of the genome is stochastic at least with regard to the

168 ultimate disposition of the silenced oma-1 and xol-1 genes at the INM.

In summary, we have shown that RNAi- and piRNA-mediated silencing trigger the

170 specialization of a $\mathrm{P}$ granule. Nuclear silencing components direct the re-localization of

171 the target genes into proximity of this specialized $P$ granule but are not required for its

172 formation. Each domain of nuage appears to serve distinct RNA targets which become

173 spatially linked to their corresponding genomic DNA targets. After the target genes

174 relocate, the nuage diminishes in size, perhaps reflecting the reduced transcriptional

175 activity of the target locus. Thus our results suggest that the transcriptional silencing 
176 machinery participates in a feedback loop that redirects the target loci and mRNA export

177 to a single specialized $\mathrm{P}$ granule. The continued low-level transcription of the paired loci

178 at the INM may serve to supply the adjacent specialized nuage with template RNA that

179 feeds small RNA amplification and inherited silencing.

180 Thousands of C. elegans germline mRNAs are targeted by nearly one million

181 distinct Argonaute guide complexes. How the system can simultaneously utilize this

182 multitude of Argonautes to surveil gene expression has been entirely mysterious. Our

183 findings suggest a partial answer to this mystery. Approximately 20 to $50 \mathrm{P}$ granules

184 surround each pachytene nucleus. Therefore, if each $\mathrm{P}$ granule is specialized to handle

185 a subset of the RNA silencing load, each P granule need only harbor 50- to 20-thousand

186 guide complexes, instead of nearly one million. Moreover by directing the co-localization

187 of their target genes each $\mathrm{P}$ granule need service only 30 to 75 of the approximately 1500

188 protein coding genes that are targeted by the system transgenerationally (Gu et al., 2009).

189 Our findings suggest that each $\mathrm{P}$ granule is initially seeded with, or differentiates

190 to contain, distinct RNA information and that $P$ granules utilize this information to guide

191 the transgenerational regulation of their target genes. During late oogenesis, some P

192 granules dissociate from germ nuclei; the dissociated $\mathrm{P}$ granules mix in the shared core

193 cytoplasm, and are taken up by enlarging oogonia (Wolke et al., 2007). P granules have

194 liquid-like properties (Brangwynne et al., 2009) and in early embryos maternally-derived

195 P granules appear to dissolve and reform in germline blastomeres (Seydoux, 2018).

196 These embryonic P granules co-localize with parentally derived Argonaute guide

197 complexes (Claycomb et al., 2009; Gu et al., 2009). Mutations that disrupt embryonic P

198 granules impair transgenerational inheritance of Argonaute silencing signals (Dodson 
199 and Kennedy, 2019; Ouyang et al., 2019). Thus it seems likely that when gene expression

200 initiates in embryonic germ cell progenitors, inherited silencing signals in these embryonic

201 P granules guide the relocalization of germline chromatin to initiate a new round of guide

202 RNA biogenesis, nuage differentiation, and chromatin re-localization.

We do not know how $\mathrm{P}$ granules compartmentalize and transmit information across

204 generations. For example, do Argonaute-guide complexes that assemble in each pachytene $\mathrm{P}$ granule, remain together, or do they become shuffled during the dynamics

206 of early embryogenesis? Similarly, the questions of how nuage becomes specialized and

207 how it orchestrates DNA relocalization will require further study. The image of nuage that

208 emerges from these studies mirrors Darwin's notion of gemmules as the units of heredity

209 (Choi and Mango, 2014; Liu, 2018) -seeds of parental information that accumulate in

210 germ cells to guide inheritance.

211 Acknowledgements: We thank Susan Strome from UC Santa Cruz, and Karen Bennett

212 from University of Missouri School of Medicine for kindly gifting reagents, Christina Baer

213 from SCOPE at UMMS for support with the micromanager installation. We thank Darryl

214 Conte, and members of the Mello and Grunwald labs for help with preparation of the

215 manuscript. This work was supported by a Howard Hughes Medical Institute

216 Investigatorship and NIH R37GM058800 (C.C.M), NSF grant 1614940 (D.G.), and NIH

217 RO1GM098583 (J.R.P). . Some of the strains used in this work were provided by the

218 Caenorhabditis Genetic Center, which is funded by the NIH National Center for Research

219 Resources (NCRR). 


\section{Methods:}

\section{Worm culture and strains:}

225 The wild-type strain N2 was used and nematodes were cultured as described(Brenner,

226 1974). The following transgenic strains were used: JJ2101: zuls242; [nmy-2p::pgl-1::gfp

$227+$ unc-119(+)] (Sheth et al., 2010), WH223: ojls9; [zyg-12::gfp + unc-119(+)], JJ2212:

228 [nmy-2p::pgl-1::mrfp]. and WM455, gfp::flag::xol-1(ne4555) (Tang et al., 2018). WM704

229 glh-1 (ne4816[gfp::g/h-1]) was generated by CRISPR genome editing as described in

230 (Ghanta et al., 2020).

232 Sample preparation for combined FISH, GFP detection and Immunofluorescence

233 General techniques.forgonad dissections were used as described (Raiders et al., 2018).

234 Briefly, worms were rinsed free of bacteria, then collected in PBS containing $0.1 \mathrm{mM}$

235 tetramisole (SIGMA L9756) as a paralytic. Once the worms stopped moving, they were

236 placed in PBS for dissection. In some preparations, $0.1 \%$ Tween-20 was used to facilitate

237 gonad transfer by micropipette. The dissected gonads were suspended briefly in buffer A

238 (75 mM Hepes (pH 6.9), $40 \mathrm{mM} \mathrm{NaCL}, 5 \mathrm{mM} \mathrm{KCL}, 2 \mathrm{mM} \mathrm{MgCL2,} 1 \mathrm{mM}$ EGTA; all

239 chemicals from Sigma), to which was added a equal volume of fixative buffer B (5\%

240 paraformaldehyde, $7.5 \mathrm{mM}$ Hepes (pH 7.2), $12 \mathrm{mM} \mathrm{NaCL}, 1.0 \mathrm{mM} \mathrm{KCL}, 0.6 \mathrm{mM}$ MgCL2,

$2410.3 \mathrm{mM}$ EGTA; all chemicals from Sigma). After fixing for $15 \mathrm{mins}$, the gonads were rinsed,

242 then incubated in RNase-free PBS (ThermoFisher AM9624) containing 0.1\% Triton-X100

243 (Sigma) for 10 mins. The gonads were transferred to room temperature $\mathrm{MeOH}$ for 10 
244 mins, then placed in fixative buffer B for an additional 5 mins and rinsed in PBS. Gonads

245 were then dehydrated and stored in $\mathrm{EtOH}$.

\section{RNA and DNA FISH}

247 Gonads stored in EtOH were rehydrated in an EtOH/PBS series (10\%, 20\%, 60\% EtOH),

248 then rinsed and incubated in two changes of wash buffer C (10\% formamide, 2 XSSC

$249[0.3 \mathrm{M} \mathrm{NaCL}, 30 \mathrm{mM}$ sodium citrate (7.0)], each rehydration and rinse step was 5 minutes.

250 Then the sample was incubated with three changes of wash buffer $\mathrm{C}$ as follows: $60^{\circ} \mathrm{C}$ for

$251 \quad 1-3 \mathrm{~min}, 70^{\circ} \mathrm{C}$ for $3 \mathrm{~min}$, followed by $60^{\circ} \mathrm{C}$ for $10-20 \mathrm{~min}$.

The wash buffer was replaced with a solution containing $\sim 0.01 \mu \mathrm{M}$ oligo probes

253 (RNA and primary DNA probes), 90\% RNA FISH bybridization buffer (Stellaris SMF-HB-

254 1), and $10 \%$ deionized formamide, and incubated at $37^{\circ} \mathrm{C}$ overnight in the dark. The

255 samples were rinsed and incubated in wash buffer $\mathrm{C}$ for 10 mins at room temperature,

256 then incubated in fresh wash buffer at $37^{\circ} \mathrm{C}$ for $1 \mathrm{hr}$. The wash buffer was removed and

257 replaced with fresh wash buffer $\mathrm{C}$ at $37^{\circ} \mathrm{C}$ for $1 \mathrm{hr}$. Gonads were rinsed and kept in PBS

258 for immunostaining experiments.

259 For combined RNA and DNA FISH experiments, the gonads were treated with an

260 additional solution containing $0.01 \mu \mathrm{M}$ Bridge oligo, $0.01 \mu \mathrm{M}$ detection oligo in 2XSSC, 30\%

261 formamide at room temperature for $3 \mathrm{hrs}$. Gonads were washed in prewarmed 2XSSC at

$26260^{\circ} \mathrm{C}$ for $20 \mathrm{~min}$, followed by two washes in 2XSSCT (2XSSC with $0.3 \%$ Triton X-100) at

$26360^{\circ} \mathrm{C}$ for $5 \mathrm{~min}$ each, and a wash in $2 \mathrm{XSSCT}$ at $60^{\circ} \mathrm{C}$ for $20 \mathrm{~min}$. Samples were then

264 rinsed at room temperature in 2XSSC, then rinsed and kept in PBS.

\section{Immunostaining}


266 After the FISH protocol was complete gonads in PBS were incubated with primary

267 antibodies diluted in PBS and 0.5 units $/ \mu$ l of RNasin (Promega N261A) at $4^{\circ} \mathrm{C}$ overnight.

268 The antibody solution was removed and the slides rinsed in three changes of PBS, 5 min

269 each. The gonads were then incubated in secondary antibodies diluted in PBS and 0.5

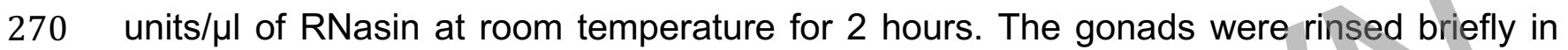

271 PBS, then immersed in mounting medium with DAPI (Vectashield H-2000) for imaging.

272 The following antibodies were used: Rabbit anti-PGL-1, a gift from Susan Strome,

273 (Kawasaki et al., 1998) Chicken anti-GLH-2, a gift from Karen Bennett (Gruidl et al., 1996).

275 Microscope settings and configuration:

276 All images were acquired on a Yokogawa CSU21 spinning disk confocal microscope

277 mounted onto an Axiovert 200M and operated with Micromanager(Edelstein et al., 2010)

278 software. The sample was mounted using a slide holder and motorized stage (ASI), and

279 images were acquired with a Zeiss 63x (1.4 NA) Planapo oil immersion objective. Optical

$280 \quad z$ stacks were collected at increments of 0.5 microns. The spinning disk unit added roughly

281 a $2.5 \mathrm{x}$ magnification factor resulting in a pixel size of $\sim 100 \mathrm{~nm}$ in the image plane. Images

282 were acquired with a Hamamatsu ImageEM (model C9100-13) series camera using an

283 EM gain of 300 and set to free read mode. Light from four lasers in a custom laser module

284 (made by Solamere) containing $405 \mathrm{~nm}, 488 \mathrm{~nm}, 561 \mathrm{~nm}$ and $640 \mathrm{~nm}$ laser lines was

285 coupled into a monomode optical fiber and delivered to the Yokogawa scan head. The

286 following bandpass filters (Chroma) were used as emission filters ET-460-50, ET-525-50,

287 ET-605-52, ET-700-75. Laser power was adjusted for each sample to minimize sample

288 bleaching. Typical acquisition times were between 400-500 msecs/frame. 


\section{Image processing:}

291 Composite images were stitched from 9 sub-images that have $50 \%$ overlapping with

292 adjacent images (Preibisch et al., 2009), but subregions were cropped to size to show

293 desired features in the final figure. Spatial offsets between color channels in lateral $(x, y)$

294 and axial ( $\mathrm{z}$ ) directions were estimated using $500 \mathrm{~nm}$ sized four color-stained beads

295 (Tetraspecks, Thermo Fisher, Catalog number T14792). The center plane is defined as

296 the plane with the largest diameter signal within a bead and was determined manually in

297 an unprocessed image stack. Raw image stacks, after axial offset correction, were filtered

298 using a Gaussian kernel with a width $\sigma=1$, a Laplacian of Gaussian (LoG3D filter (Sage

299 et al., 2005)) followed by a Gaussian kernel with a width $\sigma=1$. LoG3D is used for

300 background suppression and not to localize the exact position of a spot. The sigma along

301 the optical axis is taken as roughly half the number of slices the signal is visible in. This

302 approach preserves the visual impression of signal dimension between the channels. The

303 sigma for each channel used was: Ch1: $\sigma_{x, y}=1$ pixel, $\sigma_{z}=2$ pixel; Ch2: $\sigma_{x, y}=1$ pixel, $\sigma_{z}=$

3042 pixel; Ch3: $\sigma_{x, y}=1$ pixel, $\sigma_{z}=2$ pixel. 


\section{Figure legends:}

308 Figure 1. Pachytene germ nuclei in adult hermaphrodite gonads examined at 0,6 , and

30921 hours after exposure to oma-1 dsRNA [oma-1(RNAi)]. Single gonads are shown in the

310 paired panels $A / D, B / E, C / F, G / J, H / K$, and I/L. The larger image in each panel shows an

311 optical z-projection of about 30 planes, corresponding to approximately half the thickness

312 of the gonad. The three smaller insets show high magnifications of single optical planes

313 through individual nuclei. (A-L) Confocal images of RNA FISH, DNA FISH, and

314 fluorescent-protein signals ( $\mathrm{P}$ granule = GFP:GLH-1 and Nuclear Env =ZYG-12::GFP).

315 Signals were colored either green or magenta as indicated at top, such that coincident

316 signals appear white. For example, perinuclear RNA in panel D is magenta, but the

317 nuclear RNA appears, white because it is coincident with the green DNA signal. Before,

318 and at early timepoints in, oma-1(RNAi), the pair of nuclear foci of DNA and coincident

319 appear within a small radius around the synapsed, pachytene chromosomes, and appear

320 randomly oriented with respect to the nuclear envelope (panels A and B). By $21 \mathrm{hrs}$, a

321 single focus of DNA and coincident RNA appears juxtaposed to the envelope (panel C).

322 Prior to RNAi most cytoplasmic oma-1 mRNA in the gonad is contained in the shared

323 core region underlying the nuclei and appears as a central, vertical band in panels A, D, 324 and J.

326 Figure 2. Wild-type or mutant gonads exposed to oma-1 dsRNA for $6 \mathrm{hr}$ or $21 \mathrm{hrs}$ as

327 indicated. Images are confocal images, as in Figure 1 with signals represented in green 
328 or magenta, and merged, coincident signals in white. P granules were detected using a

329 antiserum specific for GLH-2.

331 Supplemental Figure 2. Wild-type or mutant gonads as indicated exposed to oma-1

332 dsRNA for Ohr. Images are confocal images, as in Figure 1 and 2.

334 Figure 3. (A-F) Merged confocal images as in Figures 1 and 2 of dissected gonads

335 isolated from strains that encode gfp fused to the endogenous $x 01-1$ gene. DNA and RNA

336 FISH signals were detected with both $x o l-1$ and $g f p$ specific probes (see Methods). (A-D)

337 Merged confocal images of the pachytene zones in wt (A B) and prg-1 mutants (C D).

338 Insets show single $Z$ sections of representative nuclei. ( $E$ and $F$ ) Simultaneous detection

339 of $\mathrm{P}$ granules and oma-1 and $x o l-1$ RNA $(E)$ or DNA (F) in late pachytene wildtype gonads

340 after exposure to oma-1 dsRNA for 6 hours (E) or 21 hours (F). The oma-1 and xol-1

341 signals were in different regions of the nucleus, and in general were not both visible in a

342 single focal plane, therefore the separation of these signals in individual nuclei is

343 illustrated here by a projection of optical $z$ planes through the entire nuclei (paired

344 arrowheads in $E$ and $F$ ). The colocalization of $x o l-1$ RNA and $P$ granule signal in (E) was

345 barely visible in this composite where contrast was adjusted to detect the much more

346 intense oma-1 RNA $\mathrm{P}$ granule signal. $\mathrm{P}$ granules were detected using a mixture of

347 antisera specific for GLH-2 and PGL-1.

349 Figure 4. Model illustrating events in RNA silencing. In the top panels the initiation of

350 silencing induces clearance of cytoplasmic mRNA by cytoplasmic WAGO Argonautes 
351 (yellow Argonautes in the model). By 6 hours, the RNA signal becomes limited to a single

352 P granule which expands to support the amplification of small RNAs and to coordinate

353 the programming of nuclear Argonautes (blue). Nuclear silencing in turn initiates

354 heterochromatin formation and the capture of the target genes in proximity to the adjacent

355 INM. After the target genes spatially connect to nuage, the nuage diminishes in size.

356 When the nuclear RNAi machinery is disarmed by mutation (lower panel), a single

357 enlarged $\mathrm{P}$ granule still forms, but the target genes fail to relocalize to the nuclear

358 periphery, and mRNA once again accumulates in multiple $\mathrm{P}$ granules.

References:

363 Ahringer, J., and Gasser, S.M. (2018). Repressive Chromatin in Caenorhabditis elegans: 364 Establishment, Composition, and Function. Genetics 208, 491-511. Ashe, A., Sapetschnig, A., Weick, E.M., Mitchell, J., Bagijn, M.P., Cording, A.C., Doebley, A.L., Goldstein, L.D., Lehrbach, N.J., Le Pen, J., et al. (2012). piRNAs can trigger a multigenerational epigenetic memory in the germline of C. elegans. Cell 150, 88-99.

Beliveau, B.J., Boettiger, A.N., Avendano, M.S., Jungmann, R., McCole, R.B., Joyce, E.F., KimKiselak, C., Bantignies, F., Fonseka, C.Y., Erceg, J., et al. (2015). Single-molecule superresolution imaging of chromosomes and in situ haplotype visualization using Oligopaint FISH probes. Nat Commun 6, 7147.

372 Billi, A.C., Fischer, S.E., and Kim, J.K. (2014). Endogenous RNAi pathways in C. elegans.

373 WormBook, 1-49. Julicher, F., and Hyman, A.A. (2009). Germline P granules are liquid droplets that localize by controlled dissolution/condensation. Science 324, 1729-1732. Brenner, S. (1974). The genetics of Caenorhabditis elegans. Genetics 77, 71-94. Buckley, B.A., Burkhart, K.B., Gu, S.G., Spracklin, G., Kershner, A., Fritz, H., Kimble, J., Fire, A., and Kennedy, S. (2012). A nuclear Argonaute promotes multigenerational epigenetic inheritance and germline immortality. Nature 489, 447-451. 
Chen, C.C., Simard, M.J., Tabara, H., Brownell, D.R., McCollough, J.A., and Mello, C.C. (2005). A member of the polymerase beta nucleotidyltransferase superfamily is required for RNA interference in C. elegans. Curr Biol 15, 378-383. Choi, Y., and Mango, S.E. (2014). Hunting for Darwin's gemmules and Lamarck's fluid: transgenerational signaling and histone methylation. Biochim Biophys Acta 1839, 14403881453. Claycomb, J.M., Batista, P.J., Pang, K.M., Gu, W., Vasale, J.J., van Wolfswinkel, J.C., Chaves, D.A., Shirayama, M., Mitani, S., Ketting, R.F., et al. (2009). The Argonaute CSR-1 and its 22G-RNA cofactors are required for holocentric chromosome segregation. Cell 139, 123-134.

392 Dodson, A.E., and Kennedy, S. (2019). Germ Granules Coordinate RNA-Based Epigenetic Inheritance Pathways. Dev Cell 50, 704-715 e704. Edelstein, A., Amodaj, N., Hoover, K., Vale, R., and Stuurman, N. (2010). Computer control of microscopes using microManager. Curr Protoc Mol Biol Chapter 14, Unit14 20. Fields, B.D., Nguyen, S.C., Nir, G., and Kennedy, S. (2019). A multiplexed DNA FISH strategy for assessing genome architecture in Caenorhabditis elegans. Elife 8. specific genetic interference by double-stranded RNA in Caenorhabditis elegans. Nature 391, 806-811.

401

402

403 Gruidl, M.E., Smith, P.A., Kuznicki, K.A., McCrone, J.S., Kirchner, J., Roussell, D.L., Strome, S., and Bennett, K.L. (1996). Multiple potential germ-line helicases are components of the germline-specific P granules of Caenorhabditis elegans. Proc Natl Acad Sci U S A 93, 13837-13842. Gu, W., Shirayama, M., Conte,D., Jr., Vasale, J., Batista, P.J., Claycomb, J.M., Moresco, J.J., Youngman, E.M., Keys, F., Stoltz, M.J., et al. (2009). Distinct argonaute-mediated 22G-RNA pathways direct genome surveillance in the C. elegans germline. Mol Cell 36, 231-244. Guang, S., Bochner, A.F., Burkhart, K.B., Burton, N., Pavelec, D.M., and Kennedy, S. (2010). Small regulatory RNAs inhibit RNA polymerase II during the elongation phase of transcription. Nature 465, 1097-1101. Holoch, D., and Moazed, D. (2015). RNA-mediated epigenetic regulation of gene expression. Nat Rev Genet 16, 71-84.

412 Ji, N., and van Oudenaarden, A. (2012). Single molecule fluorescent in situ hybridization (smFISH) of C. elegans worms and embryos. WormBook, 1-16.

Kawasaki, I., Shim, Y.H., Kirchner, J., Kaminker, J., Wood, W.B., and Strome, S. (1998). PGL-1, a predicted RNA-binding component of germ granules, is essential for fertility in C. elegans. Cell 94, 635-645.

Liu, Y. (2018). In Search of Darwin's Imaginary Gemmules. Adv Genet 101, 87-114.

Malone, C.J., Misner, L., Le Bot, N., Tsai, M.C., Campbell, J.M., Ahringer, J., and White, J.G. (2003). The C. elegans hook protein, ZYG-12, mediates the essential attachment between the centrosome and nucleus. Cell 115, 825-836. Ouyang, J.P.T., Folkmann, A., Bernard, L., Lee, C.Y., Seroussi, U., Charlesworth, A.G., Claycomb, J.M., and Seydoux, G. (2019). P Granules Protect RNA Interference Genes from Silencing by piRNAs. Dev Cell 50, 716-728 e716.

424 Pitt, J.N., Schisa, J.A., and Priess, J.R. (2000). P granules in the germ cells of Caenorhabditis elegans adults are associated with clusters of nuclear pores and contain RNA. Dev Biol 219, 315-333. 
429 Raiders, S.A., Eastwood, M.D., Bacher, M., and Priess, J.R. (2018). Binucleate germ cells in 430 Caenorhabditis elegans are removed by physiological apoptosis. PLoS Genet 14, e1007417. 431 Sage, D., Neumann, F.R., Hediger, F., Gasser, S.M., and Unser, M. (2005). Automatic tracking of 432 individual fluorescence particles: application to the study of chromosome dynamics. IEEE 433 Trans Image Process 14, 1372-1383.

434 Seydoux, G. (2018). The P Granules of C. elegans: A Genetic Model for the Study of RNA435 Protein Condensates. J Mol Biol 430, 4702-4710.

436 Sheth, U., Pitt, J., Dennis, S., and Priess, J.R. (2010). Perinuclear P granules are the principal 437 sites of mRNA export in adult C. elegans germ cells. Development 137, 1305-1314.

438 Shirayama, M., Seth, M., Lee, H.C., Gu, W., Ishidate, T., Conte, D., Jr., and Mello, C.C. (2012). 439 piRNAs initiate an epigenetic memory of nonself RNA in the C. elegans germline. Cell 150, 6544077.

441 Tang, W., Seth, M., Tu, S., Shen, E.Z., Li, Q., Shirayama, M., Weng, Z., and Mello, C.C. (2018). A 442 Sex Chromosome piRNA Promotes Robust Dosage Compensation and Sex Determination in 443 C. elegans. Dev Cell 44, 762-770 e763.

444 Updike, D., and Strome, S. (2010). P granule assembly and function in Caenorhabditis elegans 445 germ cells. J Androl 31, 53-60.

446 Watanabe, T., and Lin, H. (2014). Posttranscriptional regulation of gene expression by Piwi 447 proteins and piRNAs. Mol Cell 56, 18-27.

448 Weiser, N.E., and Kim, J.K. (2019). Multigenerational Regulation of the Caenorhabditis 449 elegans Chromatin Landscape by Germline Small RNAs. Annu Rev Genet 53, 289-311.

450 Wolke, U., Jezuit, E.A., and Priess, J.R. (2007). Actin-dependent cytoplasmic streaming in C. 451 elegans oogenesis. Development 134, 2227-2236.

452 


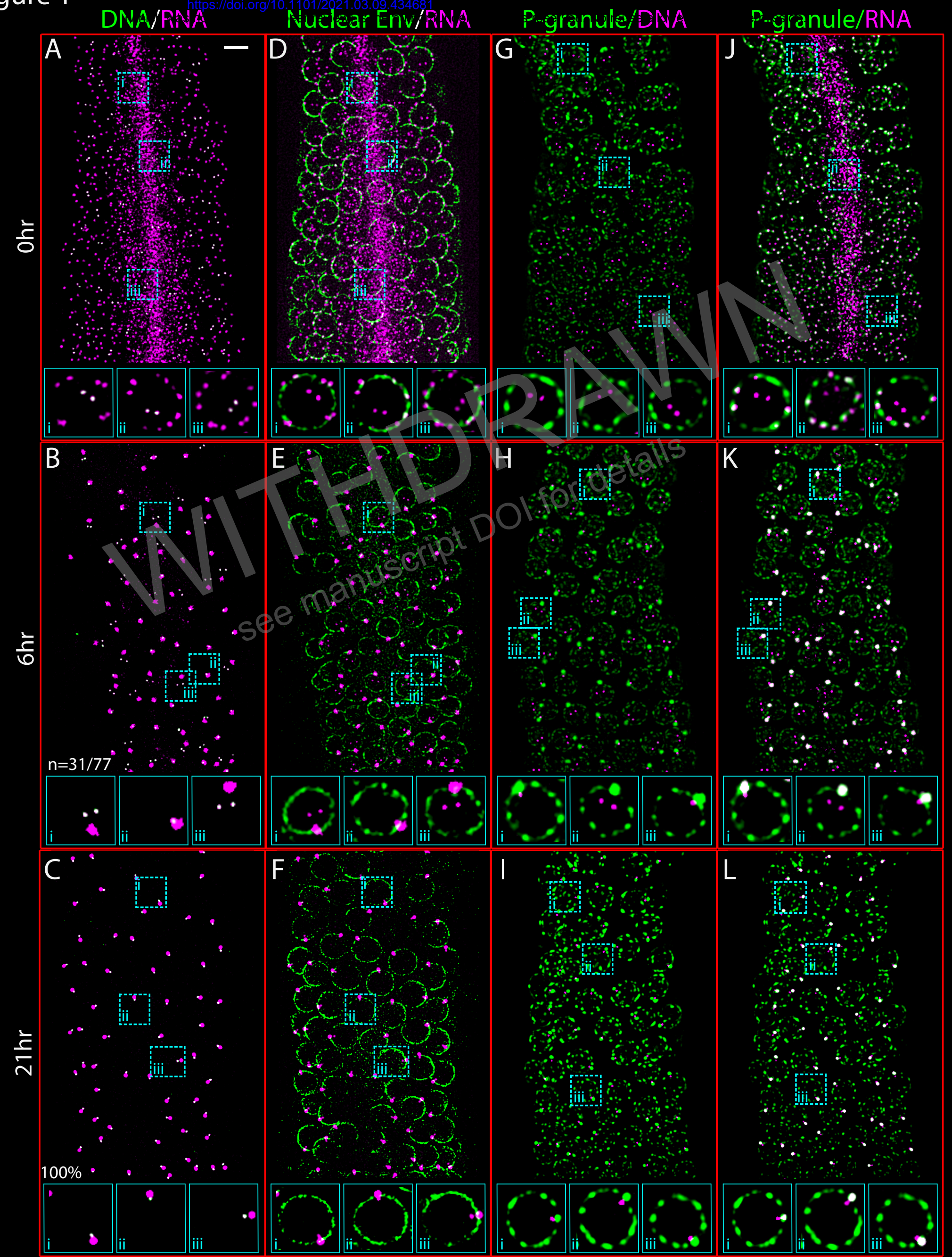

P.granule/RNA 
Figure 2

$6 \mathrm{hr}$
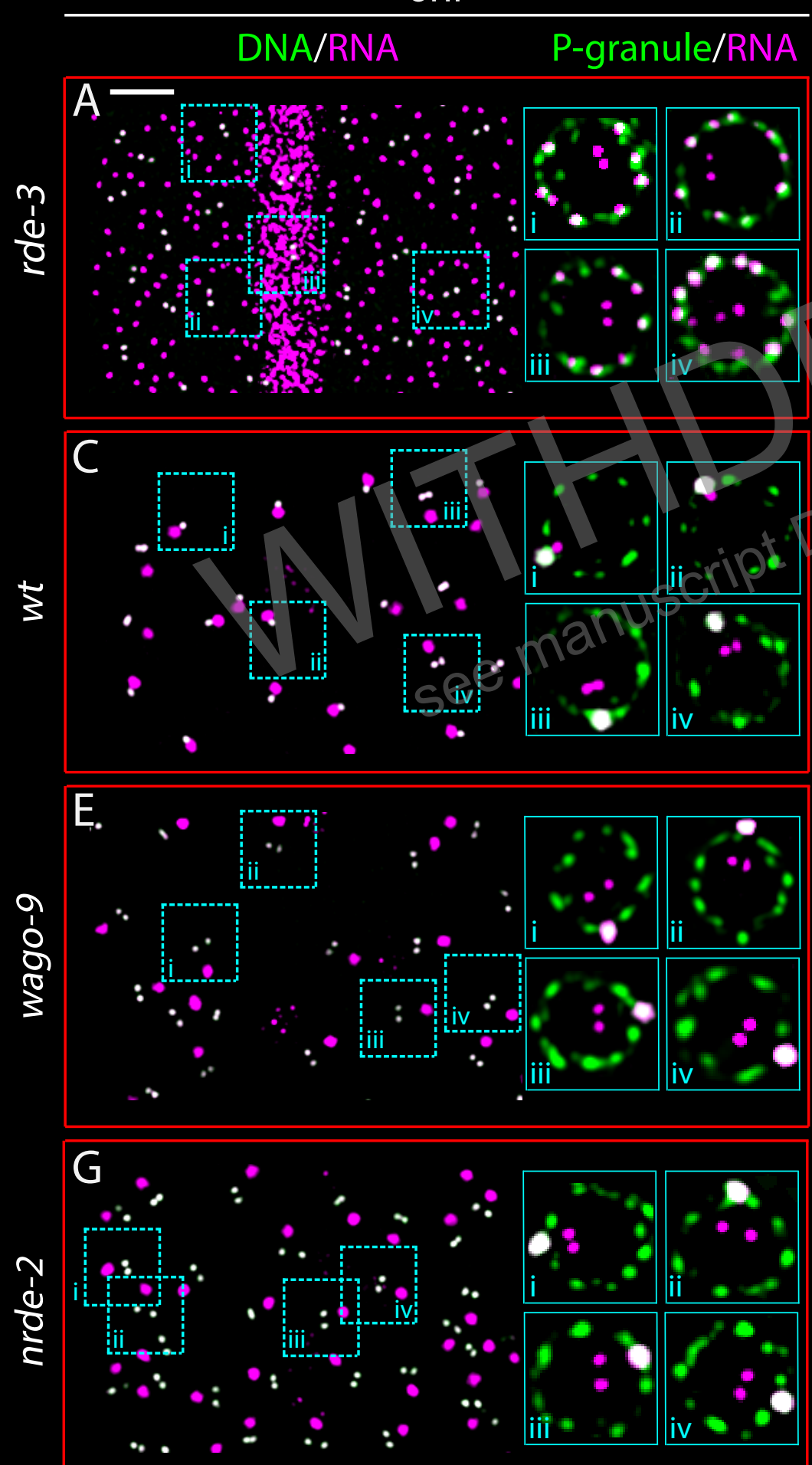

DNA/RNA

P-granule/RNA
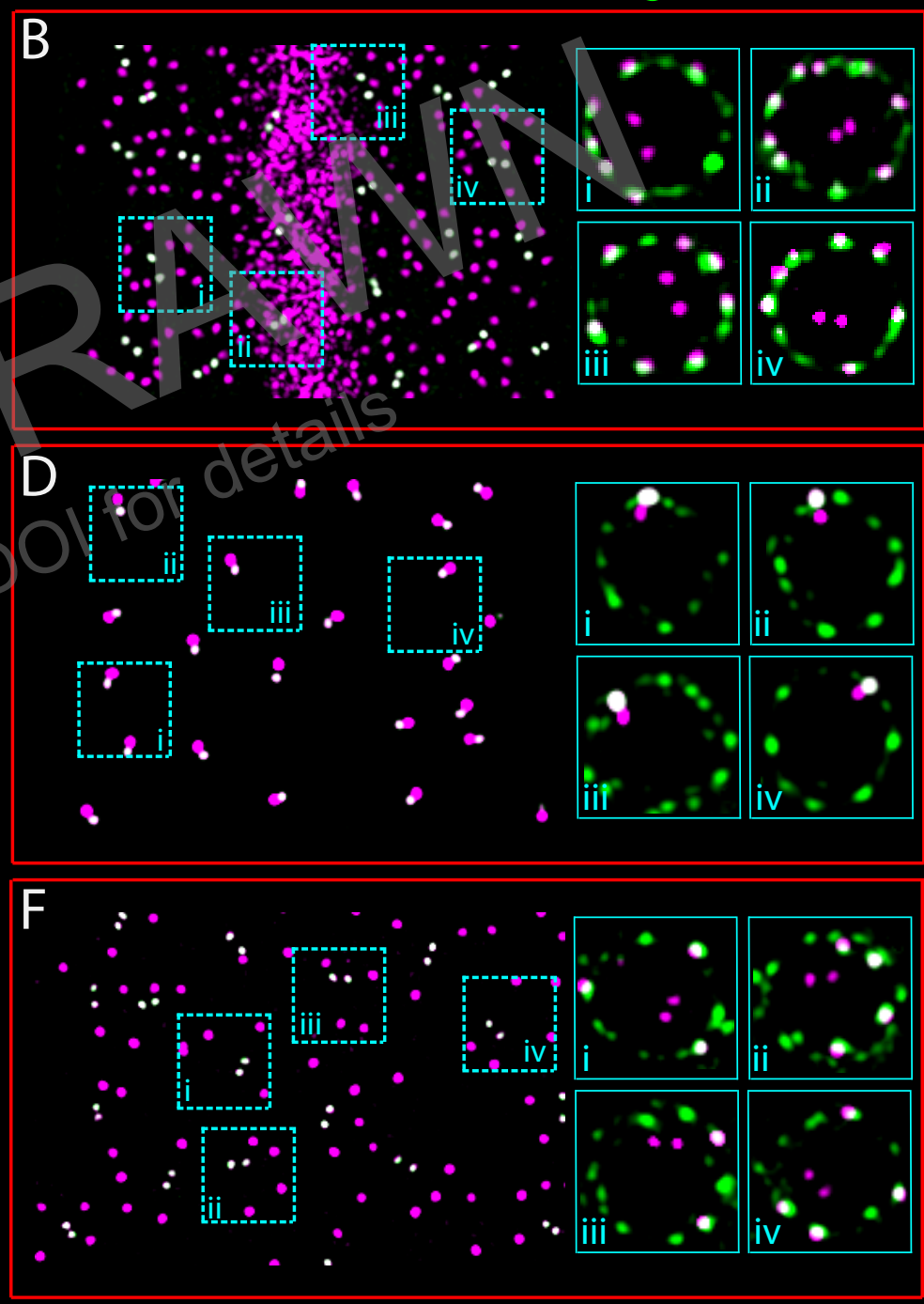

$\mathrm{H}$

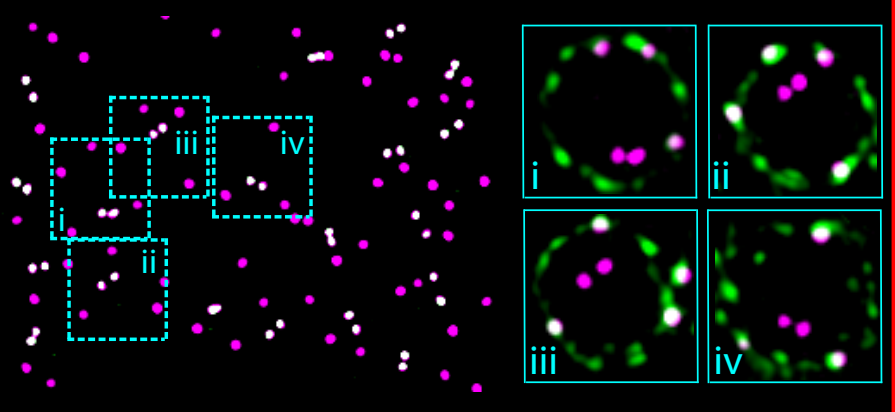




\section{Figure 3}

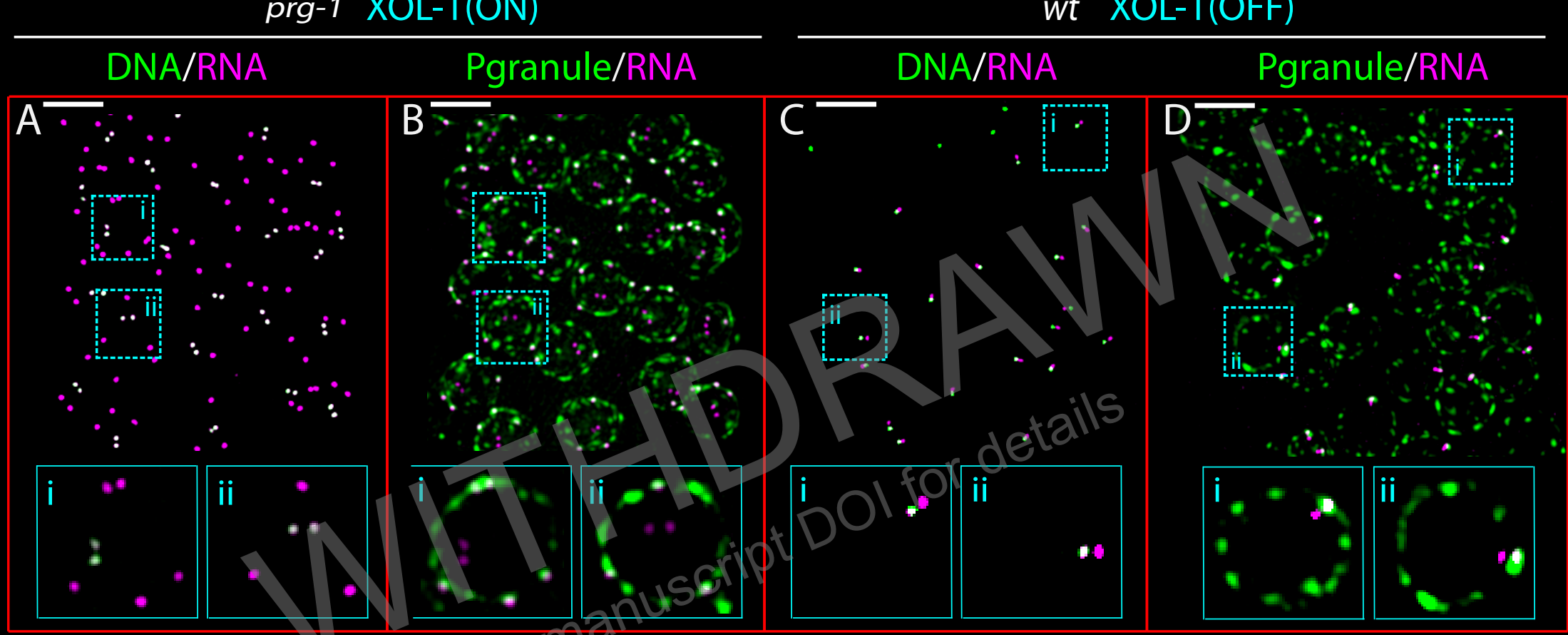

wt (6 hr): xol-1 RNA/ omagej(BNA/ Pgranule

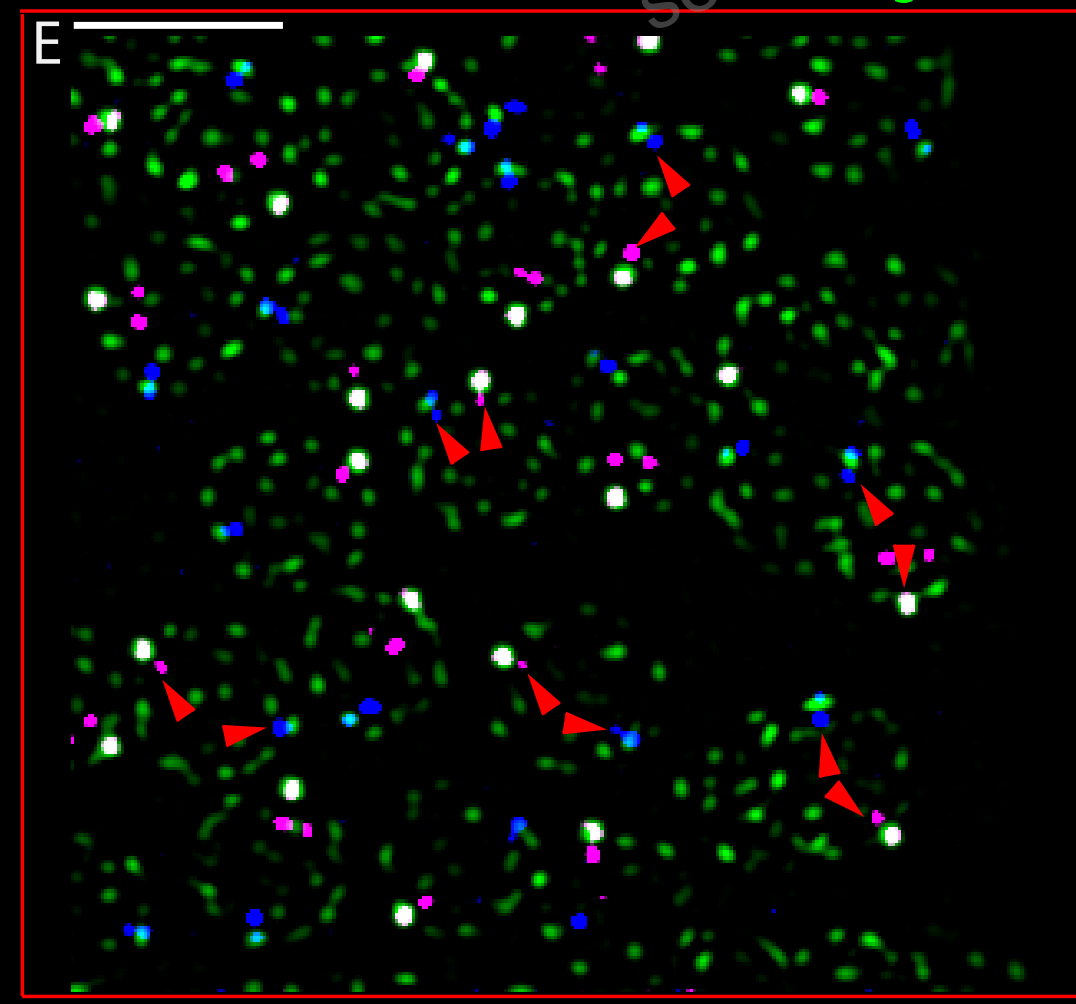

wt (21hr): xol-1 DNA/oma-1 DNA/ Pgranule

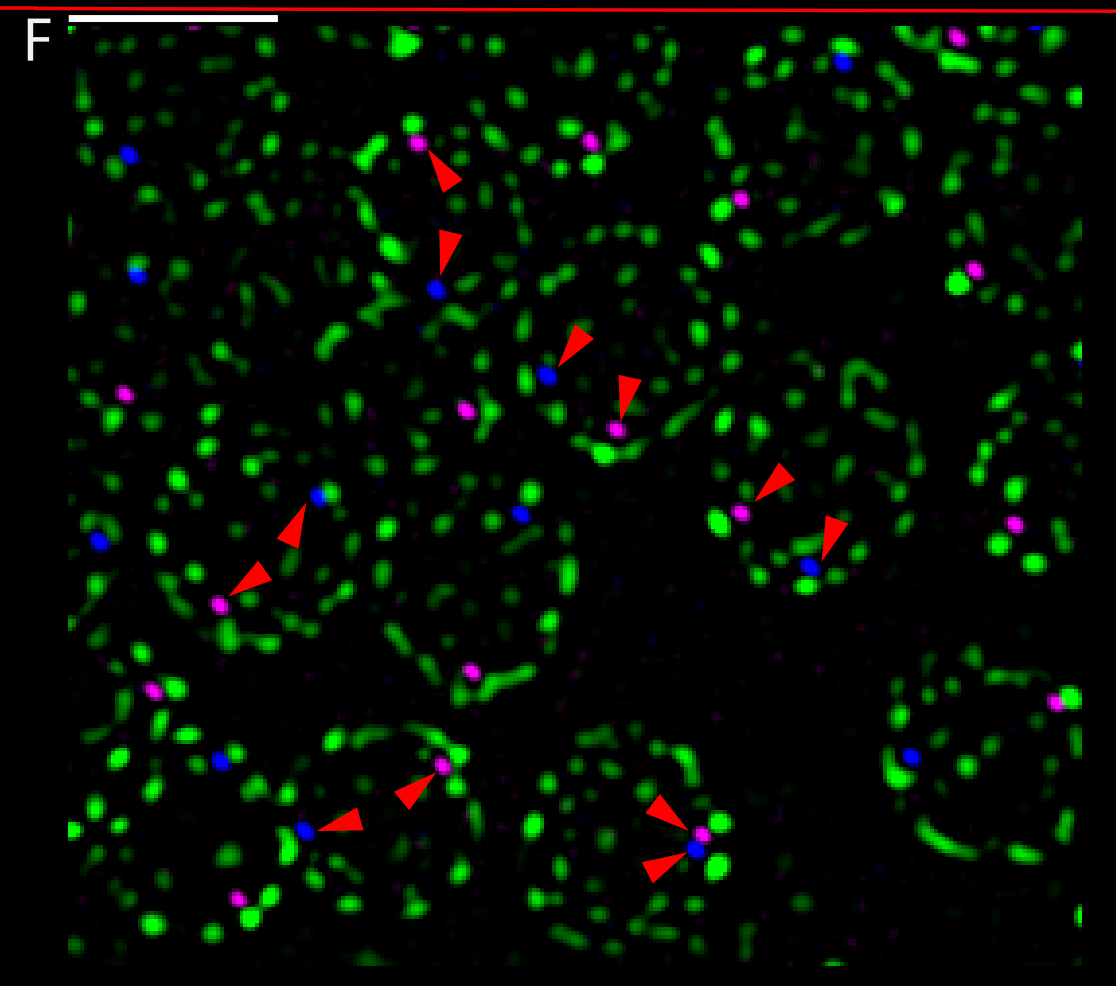




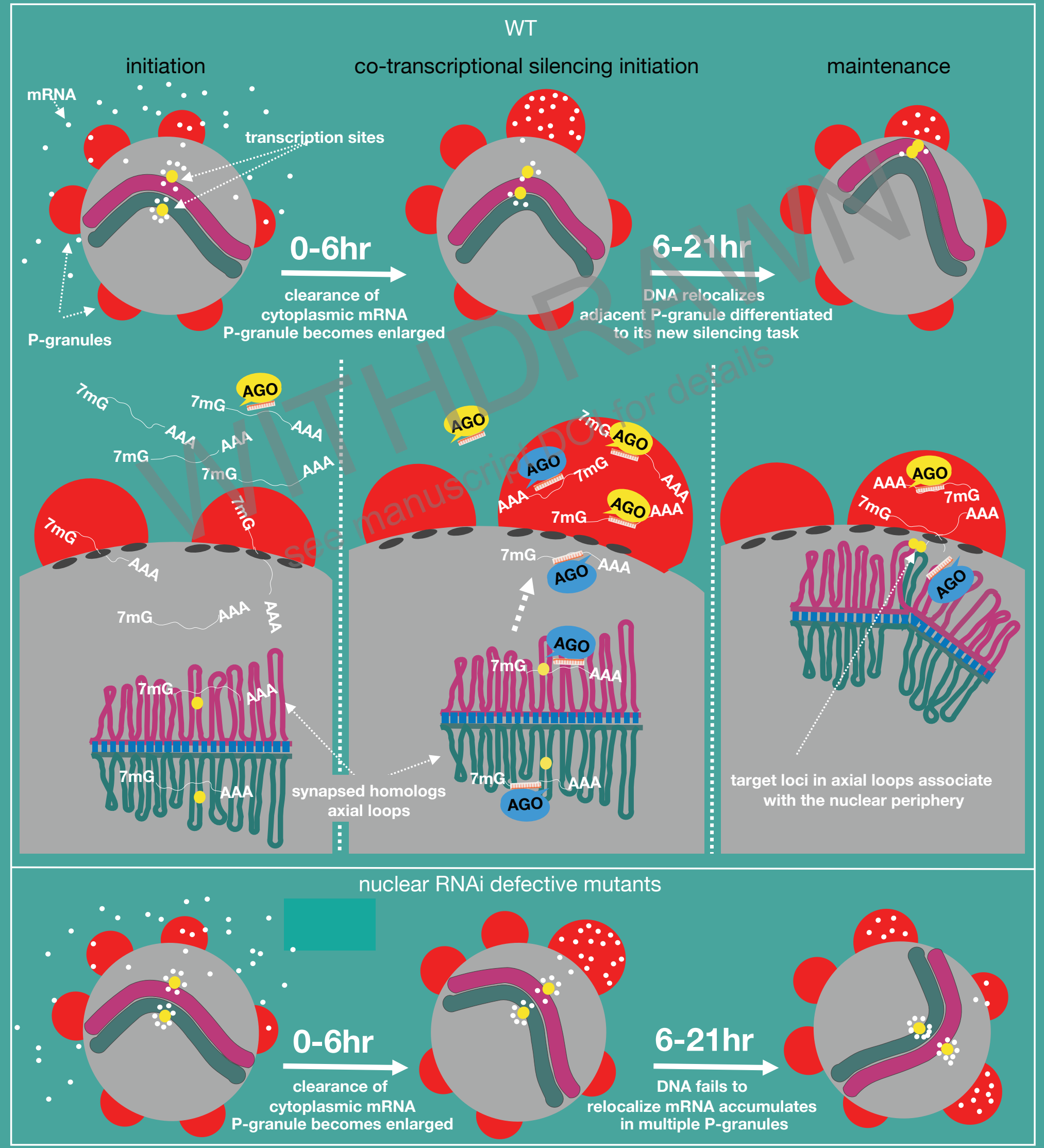


DNA/RNA

\section{A}

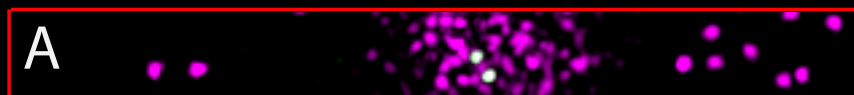

$\frac{m}{d}$
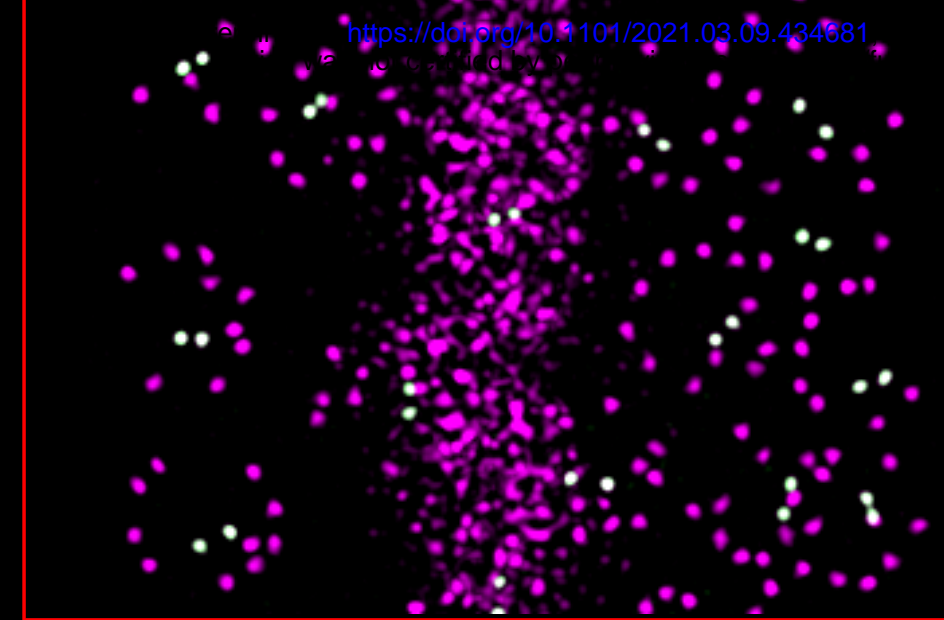

C $\because \because \cdots$ :

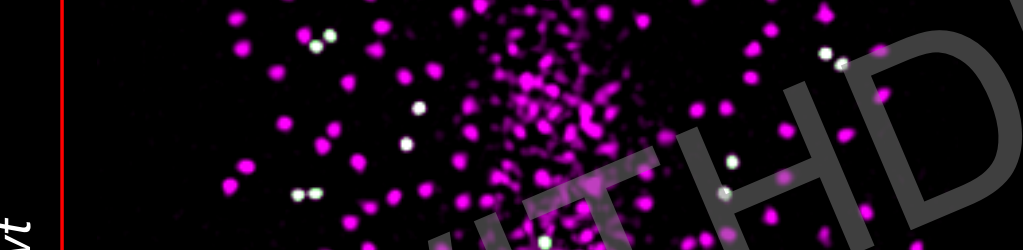

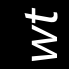

9
1
0
0
0
3

E
B

P-granule/RNA $\therefore$.

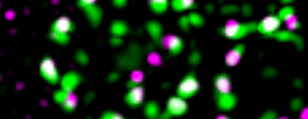

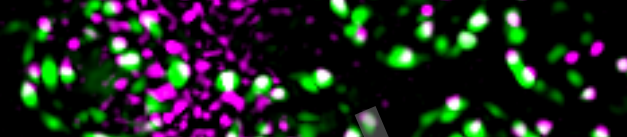

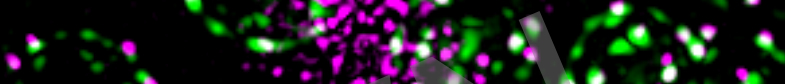

$\because \cdots \quad \%$
D एकी

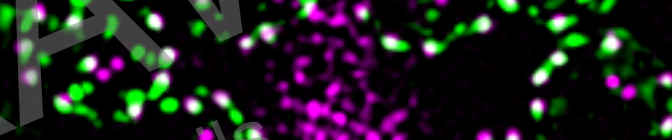
से ally $3: \cdots$ 6) $0.0 \%: 0:$

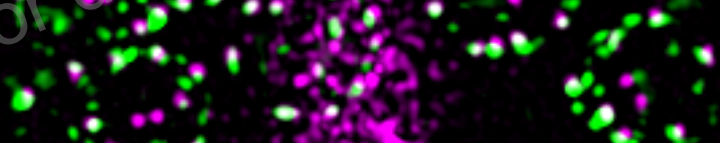
$\because \because 8 y^{2} \quad \%$

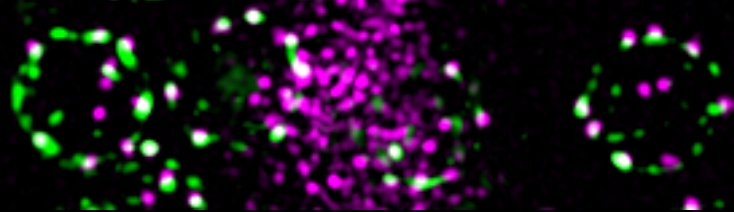
F $\because \cdots \because 8$,

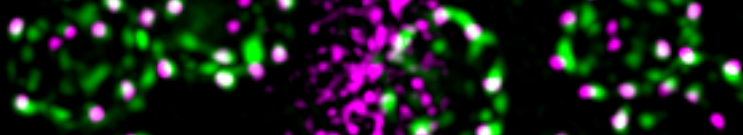

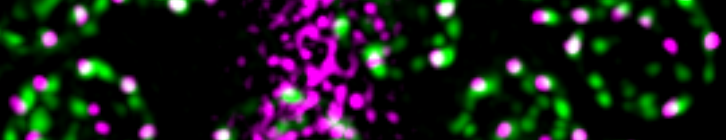

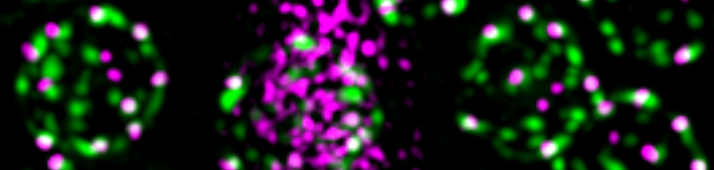

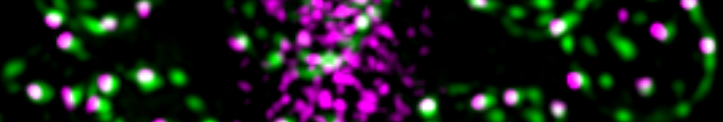

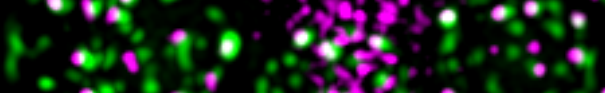

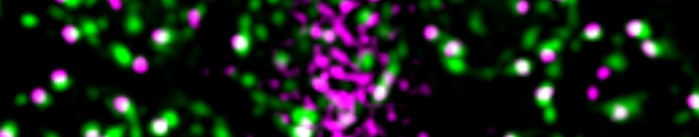
$\therefore 2+\%$

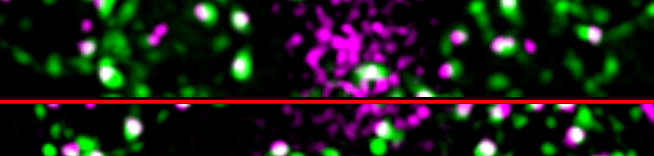
G $\cdots$ $\mathrm{H} \quad 40,8,0,0$

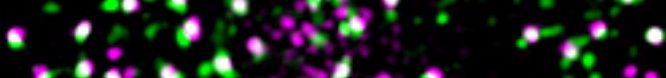

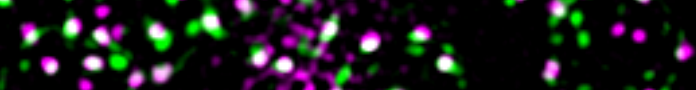
$\because 0^{\circ} \quad 38 \div$

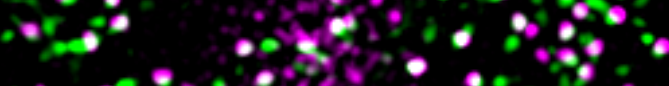

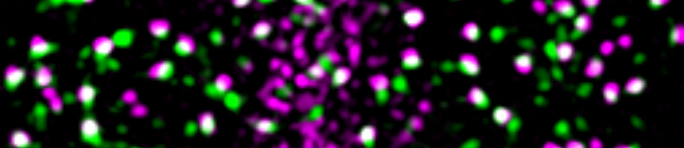

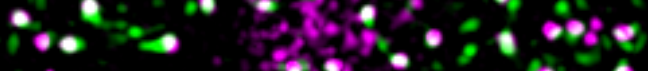

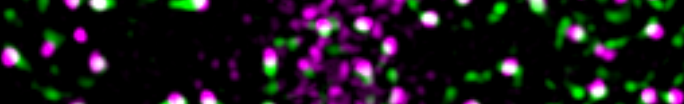

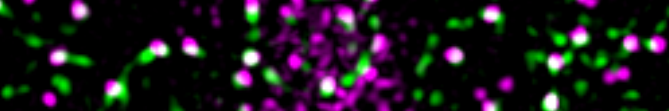

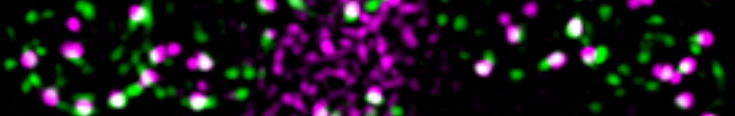

